\title{
Interfacial properties in a discrete model for tumor growth
}

\author{
Belén Moglia, ${ }^{1,2}$ Nara Guisoni, ${ }^{1}$ and Ezequiel V. Albano ${ }^{1,2}$ \\ ${ }^{1}$ Instituto de Física de Líquidos y Sistemas Biológicos (IFLYSIB), Universidad Nacional de La Plata, CONICET CCT-La Plata; \\ Calle 59 Nro 789, (1900) La Plata, Argentina \\ ${ }^{2}$ Departamento de Física, Facultad de Ciencias Exactas, Universidad Nacional de La Plata, La Plata, Argentina
}

(Received 20 September 2012; revised manuscript received 1 December 2012; published 18 March 2013)

\begin{abstract}
We propose and study, by means of Monte Carlo numerical simulations, a minimal discrete model for avascular tumor growth, which can also be applied for the description of cell cultures in vitro. The interface of the tumor is self-affine and its width can be characterized by the following exponents: (i) the growth exponent $\beta=0.32(2)$ that governs the early time regime, (ii) the roughness exponent $\alpha=0.49(2)$ related to the fluctuations in the stationary regime, and (iii) the dynamic exponent $z=\alpha / \beta \simeq 1.49$ (2), which measures the propagation of correlations in the direction parallel to the interface, e.g., $\xi \propto t^{1 / z}$, where $\xi$ is the parallel correlation length. Therefore, the interface belongs to the Kardar-Parisi-Zhang universality class, in agreement with recent experiments of cell cultures in vitro. Furthermore, density profiles of the growing cells are rationalized in terms of traveling waves that are solutions of the Fisher-Kolmogorov equation. In this way, we achieved excellent agreement between the simulation results of the discrete model and the continuous description of the growth front of the culture or tumor.

DOI: 10.1103/PhysRevE.87.032713

PACS number(s): 87.17.Ee, 87.10.Rt, 87.10.Hk, 68.35.Ct
\end{abstract}

\section{INTRODUCTION}

The study and understanding of the processes involved in tumor growth are topics of interdisciplinary interest that have recently attracted the attention of the physics community [1]. During the first stage of the process, called avascular tumor growth, the proliferation of some cells might become uncontrolled due to mutations of the regulatory genes involved. Under these conditions, the tumor receives oxygen and nutrients through its interface with the normal tissue. The subsequent large oxygen demand induces the tumor cells to produce angiogenic growth factors that stimulate the formation of blood vessels capable of supplying the required nutrients. After this second stage, known as angiogenesis, the process enters a third stage of vascular growth, which begins when the blood can reach the tumor [2].

Of course, the collective behavior of interacting living cells observed on tumor growth is a complex and intriguing phenomenon that is far from being well understood. Therefore, and in order to gain insight into the processes involved, biological and medical studies, which are mostly of an experimental nature, can be complemented with the aid of models, as is usual in physics and related sciences. For this purpose, one basically has two main approaches: (i) the formulation of continuous models by using differential equations that are solved by means of mathematical and/or numerical techniques [3-5] and (ii) a discrete approach where the dynamics is implemented at the level of individual cells [6-8]. Within this latter approach, tumor growth can be studied with the aid of well-established techniques and tools developed in the fields of statistical physics and computer simulations of complex systems. Furthermore, multiscale models simultaneously involving the above-mentioned approaches have also been formulated [9-11], e.g., nutrients and oxygen diffusion can be treated by means of mean-field differential equations, while the dynamics of cell proliferation and diffusion are undertaken in a discrete fashion.

In spite of these efforts we are far from achieving the ultimate goal of developing realistic models capable of helping not only to understand the tumor growth process but also to design adequate therapies. Therefore, it is still very useful to study minimal models capable of capturing relevant features of this rather complex system. Within this broad context, the aim of this paper is to propose a simple discrete model of avascular tumor growth that allows a detailed study of the tumor interfacial properties. In fact, during tumor growth, the maximum proliferation activity occurs at the growth interface [7,12-14], and in the avascular regime nutrients arrive mostly at the interface of the tumor. Furthermore, the dynamic scaling theory $[15,16]$ provides a powerful tool for the characterization of growth interfaces. In fact, the development of self-affine interfaces [15-18] can be rationalized in terms of critical exponents that, as in the case of second-order phase transitions [19], allow for the identification of few universality classes. Systems belonging to a given universality class then share all relevant interfacial properties independently of their intrinsic complexity. For this reason, the search and understanding of minimal models representing the above-mentioned universal behavior is a topic of permanent interest since it contributes to the identification of the underlying microscopic mechanisms responsible for the outgoing macroscopic collective behavior of the interface. In view of these advantages and usefulness, it is not surprising that the dynamic scaling theory of interfaces [15-18] has been applied to many biological systems such as tumor growth [13,20], cell cultures [21,22], bacteria colonies $[23,24]$, and so on. Also, the proposed model for the study of interfacial properties on avascular tumor growth can also be used in order to describe the developing interfaces of twodimensional in vitro cell cultures often employed by biologists as model systems for the study of cell proliferation under well-controlled experimental conditions [21,22]. Furthermore, we will also show that macroscopic quantities derived from the proposed microscopic model, such as the density profiles of growing cells and the growth velocity, can be described by means of the continuous Fisher-Kolmogorov equation.

The paper is organized as follows: In Sec. II we present descriptions of both the model for avascular tumor and cell culture growth, as well as the simulation method. Section III 
is devoted to providing a brief theoretical background for the treatment of interfaces. Results are presented and discussed in Sec. IV, and, finally, our conclusions are stated in Sec. V.

\section{DESCRIPTION OF THE MODEL}

In order to simulate the growth of tumor cells in vitro, we assume a discrete two-dimensional sample given by a square lattice of sides $D \times L$, which represents a sector of the glass substrate of Petri dishes in actual growth experiments (see Fig. 1) [21,22]. Along the $D$ direction (say, the vertical direction) we define the lines of our system and identify the spatial location of a cell in a given line by the index $i(0 \leqslant$ $i \leqslant D-1)$. Similarly, the $L$ direction defines the columns and the positions along them are identified by the index $j(0 \leqslant j \leqslant L-1)$. We take periodic boundary conditions along the $D$ direction, while for the $L$ direction, which corresponds to the tumor growth direction, the boundaries are left open. The above choice will become more clear on the definition of the tumor growth rules (see below). It is worth mentioning that a similar experimental setup, where empty (parallel) strips surrounded by cell-cultured ones are obtained by using microfabricated stencils, has been used for the study of collective cell migration patterns $[25,26]$. This methodology is inspired in the classical scratch-wound-healing experiments that also provide longitudinal fronts of cell cultures suitable for the study of interfacial patterns on cell migration [27,28].

In the proposed model, each site of the lattice with coordinates $(i, j)$ can be occupied by a cell or empty. Furthermore, the proliferation cycle will be considered in three steps: the first part of interphase ( $I_{1}$ cells) that represents the $G_{1}$ phase, the second part of interphase ( $I_{2}$ cells) that represents the synthesis $(S)$ and the $G_{2}$ phase, and the mitotic phase ( $M$ cells) [29]. So, each cell in the lattice can be in three different states, as follows: $I_{1}, I_{2}$, and $M$, as defined above. Note that the $I_{1}$ state represents a cell that has been just divided, so it is ready to evolve into the state $I_{2}$.

We further assume a homogeneous distribution of nutrients irrespective of the state of the $(i, j)$ th site. The above assumption is supported by the experimental setup used in Refs. [21,22], where concentration gradients of the nutrients
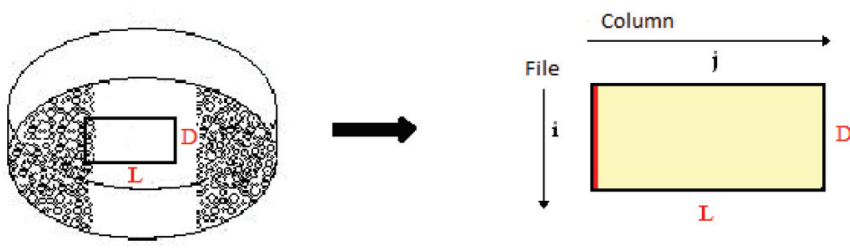

FIG. 1. (Color online) Sketches of a standard experimental setup [21] (left-hand side) and the simulation geometry used in the present work (right-hand side). A stripped stencil is (vertically) placed along a Petri dish, allowing the cell culture to proliferate up to the borders of the strip. Subsequently, the stencil is removed, setting the initial condition for the measurement of the cell culture that invades the empty space along two longitudinal fronts. See also the similar experimental setup used by Poujade et al. [25]. The rectangular geometry of our model captures a $D \times L$ sector of the culture such that a growth front develops along the horizontal $L$ axis and its interface runs along the perpendicular $D$ direction. More details in the text. are negligible. Of course, that assumption is less realistic for the case of in vivo tumor growth. The rules for the dynamic evolution of the system, which depend on the state of each particular cell, are the basis of the simulation algorithm and are defined as follows: (i) A cell of type $I_{1}$ spontaneously becomes an $I_{2}$ cell remaining at the same site. (ii) A cell in the state $I_{2}$ can either grow, becoming a cell in state $M$ with probability $P_{I}^{G}$, or diffuse into a nearest-neighbor site with probability $P_{I}^{D}=1-P_{I}^{G}$. Since an $M$ cell occupies two neighboring sites, the growth process can proceed only if a randomly selected neighboring site of the original $I_{2}$ cell is actually empty. In the same fashion, diffusion of an $I_{2}$ cell is allowed to proceed only to a neighboring empty site. (iii) Finally, a cell of type $M$ splits out into two $I_{1}$ cells, occupying the same sites as the original ones, with probability $P_{M}^{G}$. Also, this type of cell can diffuse and rotate (in steps of $\pi / 2$ degrees in the square lattice) with probabilities $P_{M}^{D}$ and $P_{M}^{R}$, with $P_{M}^{G}+P_{M}^{D}+P_{M}^{R}=1$, respectively. Of course, these two latter processes involve randomly selected neighboring sites and they are constrained by excluded volume.

In order to implement the simulations, a cell is selected at random and, depending on its state, we proceed according to the above-defined dynamic rules. After each random selection the simulation time $t_{s}$ is increased according to $t_{s}^{\text {new }}=t_{s}^{\text {old }}+$ $1 / N_{\text {tot }}$, where $N_{\text {tot }}=N_{I_{1}}+N_{I_{2}}+N_{M}$ is the total number of cells in the system. The initial condition is selected by locating $I_{1}$ cells filling the first column $(j=0)$ of the substrate. In this way, the culture can grow along the $L$ direction and a growth interface that essentially runs parallel to the $D$ direction is naturally established (cf. Fig. 1). Figure 2(a) shows typical
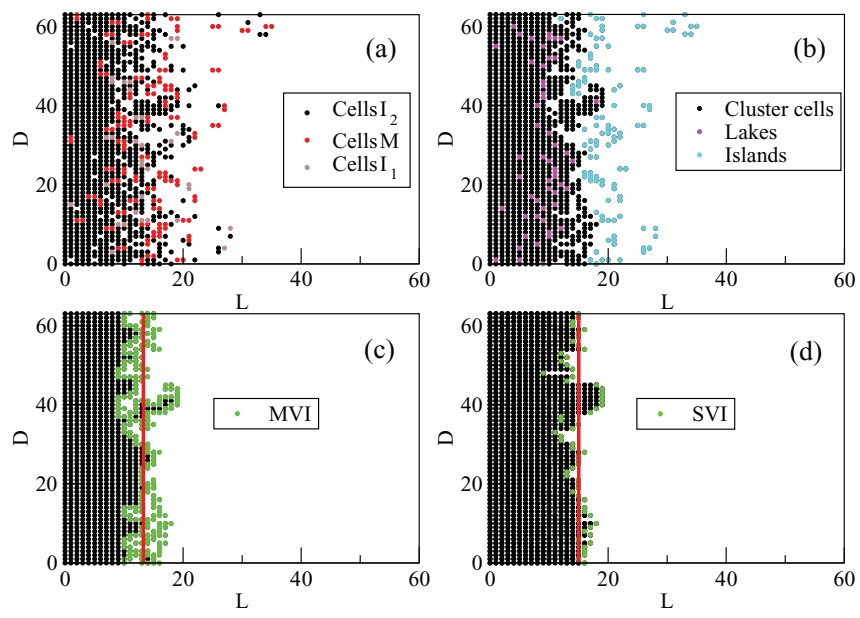

FIG. 2. (Color online) (a) Typical snapshot configuration obtained for a system of vertical side $D=64$ and for $P_{I}^{G}=P_{M}^{G}=0.10$, $P_{I}^{D}=0.90, P_{M}^{D}=0.89$, and $P_{M}^{R}=0.01$. Cells are shown by means of solid dots of different colors depending on their type (as indicated), while empty sites are white. The subsequent snapshots illustrate the procedure used to locate the interfaces of the aggregate, as follows: (b) Here, the percolating cluster linked to the initially filled column of $I_{1}$ cells, on the left-hand side of the sample, is shown by means of black dots. Also, lakes and islands are shown by means of fuchsia and turquoise dots, respectively. (c) For the sake of clarity, we remove islands and lakes and show the MVI (green dots), whose average position is shown by the vertical red line. (d) As in (c) but for the SVI. More details in the text. 
snapshot configurations that give insight into the geometry used and the shape of the growing culture.

In this paper we will focus our attention on the properties of the growth interface of the cell culture. For this purpose, it is useful to recall that there are many possible definitions of such an interface. Therefore, we will define two interfaces that are relevant for the present study, namely the so-called single-valuated interface (SVI) and the multivaluated interface (MVI), respectively. In order to locate these interfaces, one, first, determines the biggest cluster of cells (connected by means of nearest neighbors) in contact with the initially filled first column of the culture, i.e., the so-called percolation cluster [30-33], which is shown by means of black dots in Fig. 2(b). This cluster is denoted as land. On the other hand, empty sites in contact with the last column of the sample, which is never reached by the culture, are linked through both nearestand next-nearest-neighbor sites and form a large cluster that is termed the sea [notice that sites belonging to this cluster are in white in Fig. 2(b)]. The sites that are not connected with either the largest cluster of land or sea are identified as islands and lakes [see also Fig. 2(b)], respectively, but they are irrelevant. In fact, the interface is given by the seashore where land and sea are in contact [30-33], which is shown in Fig. 2(c) after removing lakes and islands. We call the set of these sites $h_{k}, k=1, \ldots, N_{\text {int }}$, the MVI, where $h_{k}$ is the horizontal coordinate ( $L$ direction, see Figs. 1 and 2 ) of the $k$ th cell belonging to the MVI, and $N_{\text {int }}$ is the number of cells of the MVI. The MVI of the snapshot depicted in Fig. 2(a) is shown in Fig. 2(c). On the other hand, for the case of the SVI, the interface has a very simple definition: after disregarding the islands, one has to follow a single line of the sample and by starting from the sea, the first site of land found is just a site of the SVI (for a similar definition see Ref. [33]), as shown in Fig. 2(d). Therefore, the SVI is given by the set of sites $\left(h_{k}, k=1, \ldots, N_{\text {int }}=D\right)$. Note that for the MVI one may have $N_{\text {int }} \geqslant D$. For further technical details on the method used for the location of the interfaces, see, e.g., Refs. [30-33].

It is worth mentioning that models similar to that studied here have been proposed, see, e.g., Smolle et al. [34] and references therein. However, these early studies are mostly of a qualitative nature and no attempts have been made to fully characterize the interfacial properties by using scaling methods.

\section{BRIEF THEORETICAL BACKGROUND.}

A useful tool for the characterization of interfaces is the well-established dynamic scaling theory developed by Family and Vicsek [15-17]. The methodology relies on the evaluation of the interface width $(W)$ and its rationalization in terms of exponents, which in turn may define a universality class. So, as in the study of second-order phase transitions [35], a set of different models sharing the same critical exponents are said to belong to the same universality class.

In order to define the interface width, first, one needs to identify the cells located at such an interface. Let $h_{k}(0 \leqslant$ $h_{k} \leqslant L-1$ and $1 \leqslant k \leqslant N_{\text {int }}$ ) be the horizontal coordinate ( $L$ direction; see Figs. 1 and 2 ) of the $k$ th cell belonging to the interface, where $N_{\text {int }}$ is the number of cells belonging to the interface. We then define the average interface position $\langle h\rangle$ as the mean value of $h_{k}$, namely

$$
\langle h\rangle=\frac{1}{N_{\text {int }}} \sum_{k=1}^{N_{\text {int }}} h_{k},
$$

where for the SVI one has $N_{\text {int }}=D$ while for the MVI one may have $N_{\text {int }} \geqslant D$. Now the interface width can be evaluated as the rms deviation of $\langle h\rangle$, namely

$$
W=\sqrt{\frac{1}{N_{\text {int }}} \sum_{k=1}^{N_{\text {int }}}\left(h_{k}-\langle h\rangle\right)^{2}} .
$$

According to the experience gained in the study of growth interfaces $[15,16]$, by starting the measurements from a flat interface, as in our case, which assumes a line of cells located in the first column of the sample as the initial condition, one has that, at early times, the width increases according to

$$
W \propto t^{\beta},
$$

where $\beta$ is the growth exponent. During the growth process it is expected that correlations between cells will start to develop. In particular, one has that correlations along the interface can be described by a typical correlation length $\left(\xi_{\|}(t)\right)$, which in turn grows,

$$
\xi_{\|}(t) \propto t^{\frac{1}{z}},
$$

where $z$ is the dynamic exponent. The above paragraphs essentially outline the expected dynamic behavior of a growth interface in an infinite sample. However, both in the experiments and in our simulations, one has to deal with finite samples of a given width (say $D$, as shown in Figs. 1 and 2). Therefore, $\xi_{\|}$cannot grow indefinitely, as stated by Eq. (4), but now it grows only up to a given crossover time $\left(t_{c}\right)$ according to

$$
\xi_{\|}\left(t_{c}\right) \propto D \propto t_{c}^{\frac{1}{2}} .
$$

Furthermore, as $t \rightarrow t_{c}$, one also observes deviations from the prediction of Eq. (3) since now the growth of the interface width becomes slower, and eventually it fluctuates around some saturation (or stationary) value $\left(W_{s}\right.$, for $\left.t>t_{c}\right)$.

Now, in order to give a unified description of the aboveoutlined arguments as in the case of second-order phase transitions $[35,36]$, the dynamic scaling theory states that the interface width is a homogeneous function of two characteristic lengths $\left(W\left(D, \xi_{\|}\right)\right)$, namely $\xi_{\|}$, which reflects the properties of the system under study, and $D$, which is set by the experimental or simulation conditions. The following scaling ansatz then can be formulated:

$$
W\left(D, \xi_{\|}\right)=W(D, t)=t^{\beta} f\left(\frac{D}{\xi_{\|}}\right)=t^{\beta} f^{*}\left(\frac{D}{t^{\frac{1}{z}}}\right),
$$

where $f^{*}(u)$ is a suitable scaling function. In particular, one has that

$$
f^{*}(u)=\left\{\begin{array}{ll}
\text { const, } & u \rightarrow \infty \\
u^{\alpha} & u \rightarrow 0
\end{array},\right.
$$

where $\alpha$ is the roughness exponent. In this way, in the early time limit $t \rightarrow 0$ (i.e., $u \rightarrow \infty$ ), one recovers Eq. (3), while in the long-time regime $t \rightarrow \infty$ (i.e., $u \rightarrow 0$ ), one has that 
the interface width becomes saturated at $D$-dependent values according to

$$
W_{s}(D) \propto D^{\alpha}
$$

where the above relationship is accomplished when $z=\frac{\alpha}{\beta}$, so only two of the exponents are actually independent.

In a more general fashion, the scaling behavior of the interface can also be investigated by evaluating the structure factor $S(q, t)$ or power spectrum [16,18,21,22,37]

$$
S(q, t)=\left\langle\left|\frac{1}{\sqrt{N_{\mathrm{int}}}} \sum_{n=1}^{N_{\mathrm{int}}} h_{n}(t) e^{-i q n}\right|^{2}\right\rangle,
$$

where $q=2 \pi n / N_{\text {int }}$, with $n=1, \ldots, N_{\text {int }} / 2$. Now a quite general dynamic scaling hypothesis can also be formulated for the structure factor, yielding [18]

$$
S(q, t)=q^{-(2 \alpha+1)} s\left(q t^{1 / z}\right),
$$

where the scaling function has the general form

$$
s(u) \sim\left\{\begin{array}{ll}
u^{2\left(\alpha-\alpha_{s}\right)} & \text { if } u \gg 1 \\
u^{2 \alpha+1} & \text { if } u \ll 1
\end{array},\right.
$$

where $\alpha_{s}$ is the spectral roughness exponent, which can only be evaluated by measuring the structure factor [18]. For the sake of completeness, one can make use of

$$
W^{2}(D, t)=\frac{1}{D} \sum_{q} S(q, t)
$$

in order to obtain a relationship between the structure factor and the width of the interface [cf. Eqs. (2) and (6)].

As already mentioned, the set of exponents that characterize a given interface determines its universality class. Within this context, it is worth mentioning some relevant examples, e.g., in $(1+1)$ dimensions the Kadar-Parisi-Zhang (KPZ) universality class gives $\alpha=1 / 2, \beta=1 / 3$, and $z=3 / 2$. The first studied model belonging to the KPZ universality class is most likely the Eden model [38], which was proposed for the description of the growth of compact tumors or bacteria colonies. Very recently, Huergo et al. [21,22] have shown that the growth interface of in vitro cellular cultures of Vero (African green monkey kidney) cells belongs to the KPZ universality class, since they have determined $\alpha=0.50(5)$ and $\beta=0.33(2)$ for essentially two-dimensional cultures performed in Petri dishes [21,22]. Also, the interface of a model for tumor growth proposed by Drasdo et al. [6,7,39] exhibits interfacial properties consistent with the KPZ universality class.

On the other hand, Brú et al. [13] claimed that a large variety of cultures, as well as two-dimensional slices of real three-dimensional tumors, may belong to the molecular beam epitaxy (MBE) universality class since they determined the following exponents $\alpha=1.5 \pm 0.15(\alpha=3 / 2)$, $\beta=0.38 \pm 0.07(\beta=3 / 8)$, and $z=4.0 \pm 0.5(z=4)$, where the figures between brackets are the exactly known values of the exponents $[16,40]$. The MBE universality class was originally found in the field of condensed matter physics, more specifically in a model for the MBE of thin solid films. The claims of Brú et al. [13] on the universality class of their samples originated a long-standing controversy [41,42] that still remains open, e.g., Block et al. [6] have suggested that the experiments of Brú et al. [13] should be reanalyzed and improved by tracking the path of marked cells in order to test the role of surface diffusion that is a key ingredient to yield MBE features.

\section{RESULTS AND DISCUSSION}

We simulated the growth of cell colonies, as described in Sec. II, on samples of different sizes, e.g., $1000 \leqslant L \leqslant 10000$ and $64 \leqslant D \leqslant 512$, while averages are taken over a number $n_{s}$ of different samples, where typically one has $1000 \leqslant$ $n_{s} \leqslant 5000$. Hereafter, we use $P_{I}^{G}=P_{M}^{G}, P_{I}^{D}=1-P_{I}^{G}$, $P_{M}^{D}+P_{M}^{R}=P_{I}^{D}$, and $P_{M}^{R}=0.01$.

Figure 3(a) shows plots of the average position of the growth interface $\langle h\rangle$ versus time as obtained for different values of the growth probability $P_{I}^{G}$. The straight lines obtained indicate that our interfaces grow at constant speeds and, from the slopes, one can determine the growth velocity $\left(v_{g}\right)$, as shown in Fig. 3(b).
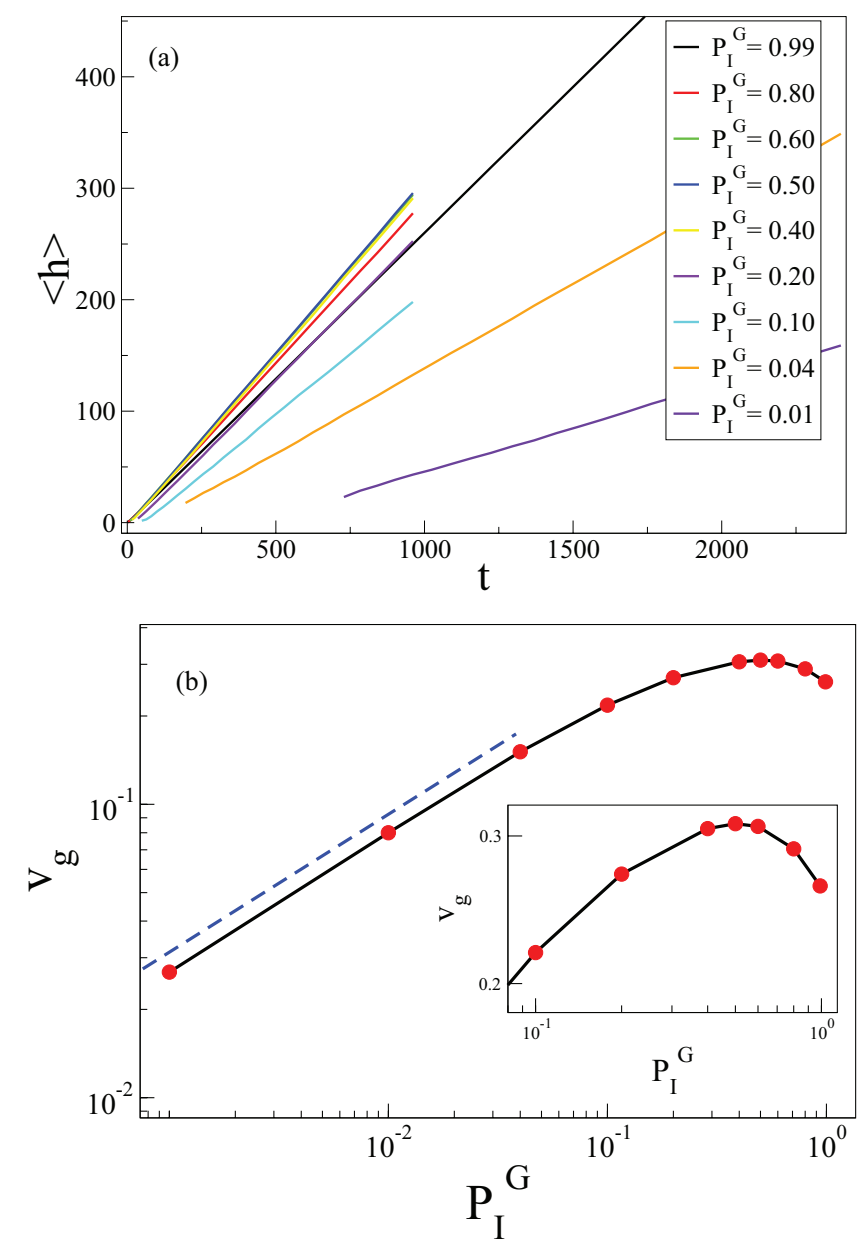

FIG. 3. (Color online) (a) Plots of the average position of the growth interface $\langle h\rangle$ versus time as obtained for different values of the growth probability $P_{I}^{G}\left(0.01 \leqslant P_{I}^{G} \leqslant 0.99\right)$, as indicated. (b) Log-log plots of the growth velocity $\left(v_{g}\right)$ versus $P_{I}^{G}$ as obtained from the slopes of the straight lines shown in (a). The dashed line (slightly shifted up for the sake of clarity) has slope $\gamma=0.47(4)$ as follows from the best fit of the data obtained according to Eq. (13) and for $P_{I}^{G}<0.10$. The inset shows a zoom of the region where $v_{g}$ exhibits a maximum. More details in the text. 
It is worth mentioning that a linear dependence in plots of $\langle h\rangle$ versus time has been reported for the propagation front of Vero colonies cultured in Petri dishes, as obtained by using two different experimental procedures [21]. Also, for some special experimental conditions, the steady wound edge advancement in crowded cultures has been reported [27]. Furthermore, our simulation results show that for low values of $P_{I}^{G}\left(P_{I}^{G}<0.1\right)$, i.e., when diffusion plays a key role in the overall behavior of the culture ( since $P_{I}^{D}=1-P_{I}^{G}$ ), the log-log plot of $v_{g}$ versus $P_{I}^{G}$ [Fig. 3(b)] yields a straight-line behavior that is consistent with a power-law dependence of the form

$$
v_{g} \propto P_{I}^{G \gamma}
$$

where, from the best fit of the data, the exponent $\gamma=0.47(4)$ is obtained, i.e., a figure that suggests that $\gamma=1 / 2$ exactly should hold [see the dashed line in Fig. 3(b)]. By increasing $P_{I}^{G}$ one observes that $v_{g}$ still grows but with a monotonically decreasing slope until it reaches a maximum value just for $P_{I}^{G}=1 / 2$ [see the inset of Fig. 3(b)] and subsequently $v_{g}$ decreases slightly. This behavior is consistent with an optimal growth whose maximum speed is achieved when the growth and diffusion probabilities are the same $\left(P_{I}^{G}=P_{I}^{D}=1 / 2\right)$.

Figures 4(a), 4(b), and 4(c) show log-log plots of the interface width $(W)$ versus time as obtained for samples of different sizes (as specified in the plots) and growth probabilities given by $P_{I}^{G}=0.99,0.50$, and 0.10 , respectively. In this way, we selected three representative growth probabilities within the range of maximal growth velocity, which lies in the range of Fig. 3(b) that is amplified in the corresponding inset.

It is worth mentioning that Fig. 4(a) combines results obtained during the early time regime $\left(t<2 \times 10^{3}\right)$ and the stationary regime $\left(t>2 \times 10^{3}\right)$, which were obtained by means of different simulation runs in order to save CPU time and achieve better statistics. For the cases $P_{I}^{G}=0.99$ and $P_{I}^{G}=0.50$ we show results obtained by measuring the SVI, which coincide with those corresponding to the MVI, since the formation of overhangs is negligible. On the other hand, for $P_{I}^{G} \leqslant 0.10$ when cell diffusion becomes large enough, one observes the formation of overhangs and clusters of cells detached from the main culture that may be incorporated due to the growth process (see, e.g., the snapshots of Fig. 2). Under these circumstances the SVI does not provide a realistic description of the system, so we report results obtained by measuring the MVI.

In all cases shown in Fig. 4 we observed an initial increase of $W$ according to equation (3), which is followed by a crossover behavior to a saturation value, as already expected according to the discussion of Eq. (8) in Sec. III. Then, by fitting the early time behavior of the interface width, we obtained $\beta=0.32(2)$ for all studied values of $P_{I}^{G}$ [see the dashed lines in Figs. 4(a)-4(c)]. On the other hand, since in order to obtain a reliable value of the roughness exponent by using Eq. (8) one needs additional simulations performed with even larger lattices than those already shown in Fig. 4, we evaluated the structure factor $S(q, t)$ [Eq. (9)] for the stationary regime of the interface, as shown in Fig. 5. In the stationary regime, of course, the structure factor is independent of time [18], and it scales as

$$
S(q, D) \propto q^{-\left(2 \alpha_{s}+1\right)} D^{2\left(\alpha-\alpha_{s}\right)} .
$$
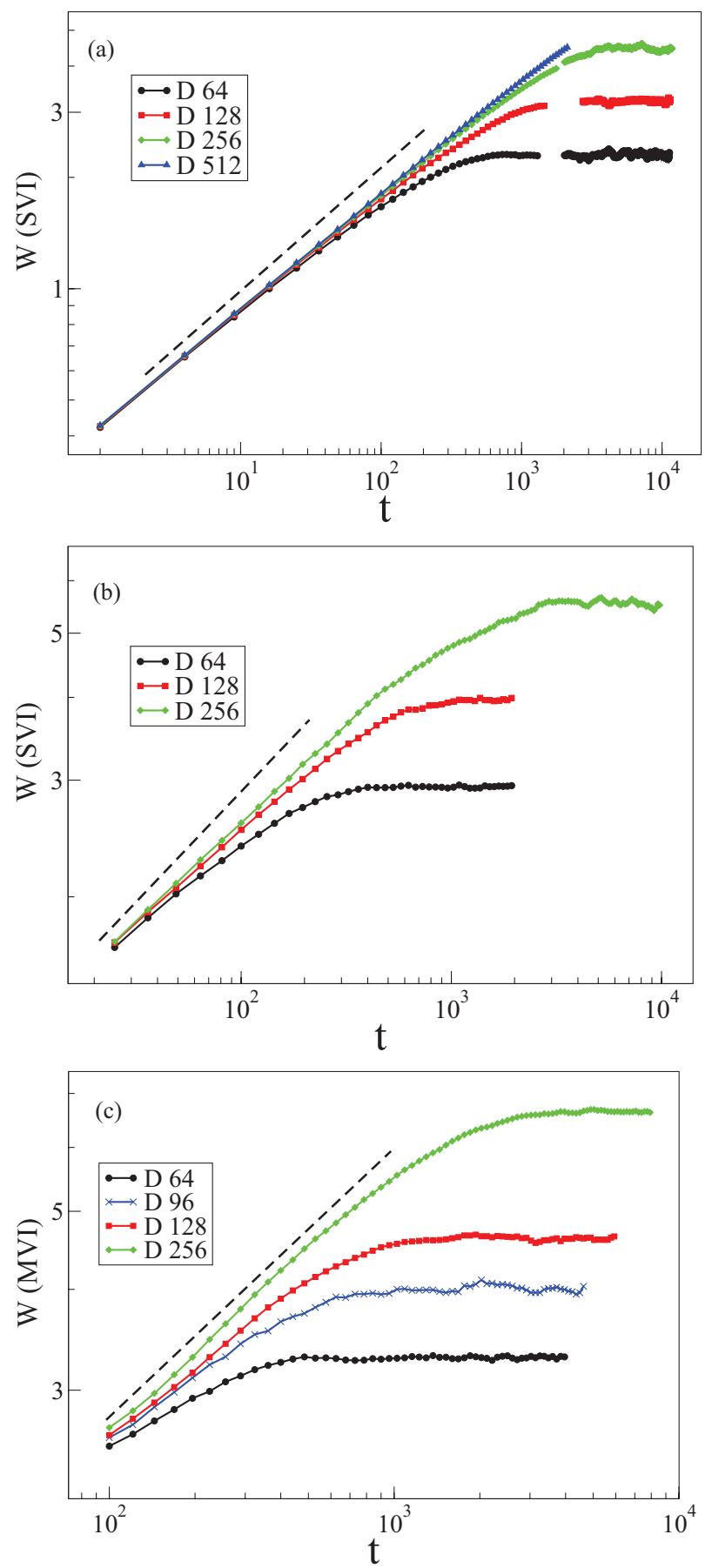

FIG. 4. (Color online) Log-log plots of the interface width $(W)$ versus time as obtained for different sample sizes, as indicated, and for different sets of parameters, as follows: (a) $P_{I}^{G}=0.99$, (b) $P_{I}^{G}=$ 0.50 , and (c) $P_{I}^{G}=0.10$. For the cases $P_{I}^{G}=0.99$ and $P_{I}^{G}=0.50 \mathrm{we}$ show results obtained by measuring the SVI, while for $P_{I}^{G}=0.10, W$ is evaluated by using the MVI. The dashed lines have slopes $\beta=1 / 3$ and have been drawn for the sake of comparison. More details in the text.

In Fig. 5(a) we observe that $S(q)$ is independent of $D$, so $\alpha=\alpha_{s}$. Also, from the best fit of the data we obtained $-\left(2 \alpha_{s}+\right.$ $1)=-1.965(50)$, i.e., $\alpha_{s}=\alpha=0.482(25)$. As discussed in Ref. [18], if one has that $\alpha=\alpha_{s}$, then the structure factor exhibits nonanomalous behavior [cf. Eqs. (10) and (11)], i.e., 

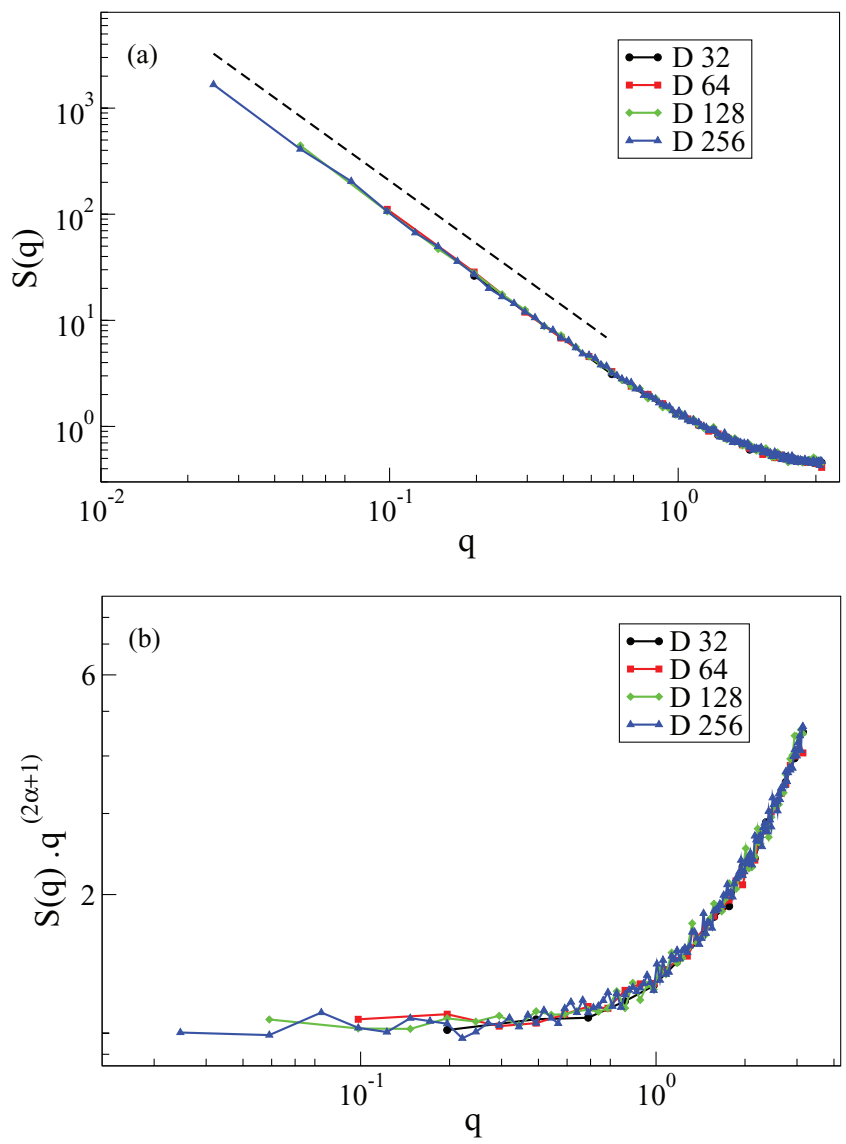

FIG. 5. (Color online) (a) Log-log plots of the structure factor $S(q)$ versus $q$ as obtained during the saturation regime of the interface. Data obtained for different lattice sizes as indicated and taking $P_{I}^{G}=$ 0.99 . Results averaged over 1000 different samples. The dashed line is the best fit of the data and it has slope $-(2 \alpha+1)=-1.965$. (b) Scaling plot of the data already shown in (a) according to Eqs. (10) and (11) and obtained by taking $\alpha=1 / 2$. More details in the text.

a characteristic feature of the KPZ universality class. Also, the scaling behavior of $S(q)$, as expected according to Eq. (14), is nicely verified by our data [cf. Fig. 5(b)].

After determining both $\beta$ and $\alpha$, we are ready to test the dynamic scaling ansatz given by Eq. (6), since one should obtain a data collapse of curves corresponding to different sample sizes just by plotting $W / D^{\alpha}$ versus $t / D^{z}$ at $\log -\log$ scales. These data collapses are shown in Figs. 6(a), 6(b), and $6(\mathrm{c})$, as obtained for $P_{I}^{G}=0.99,0.50$, and 0.10 , respectively. It is worth mentioning that the best collapses are obtained by assuming the exponents corresponding to the KPZ universality class, namely $\alpha=1 / 2, \beta=1 / 3$, and $z=3 / 2$, as suggested by our previous evaluation of the exponents from Figs. 4 and 5. Also, other universality classes, such as $\operatorname{MBE}(\alpha=3 / 2, \beta=3 / 8, z=4)$ [40] and Edwards-Wilkinson ( $\alpha=1 / 2, \beta=1 / 4, z=2$ ) [43], can safely be disregarded. Our findings then agree with the exponents determined for in vitro cultures of Vero colonies, namely $\alpha=0.50(5)$ and $\beta=0.33(2)[21,22]$. On the other hand, the onset of interfacial roughening of an initially homogeneous culture has been observed for the collective motion of both an epithelial monolayer [25] and brain tumor cells [28], but no attempts to determine the roughness exponents has been reported.
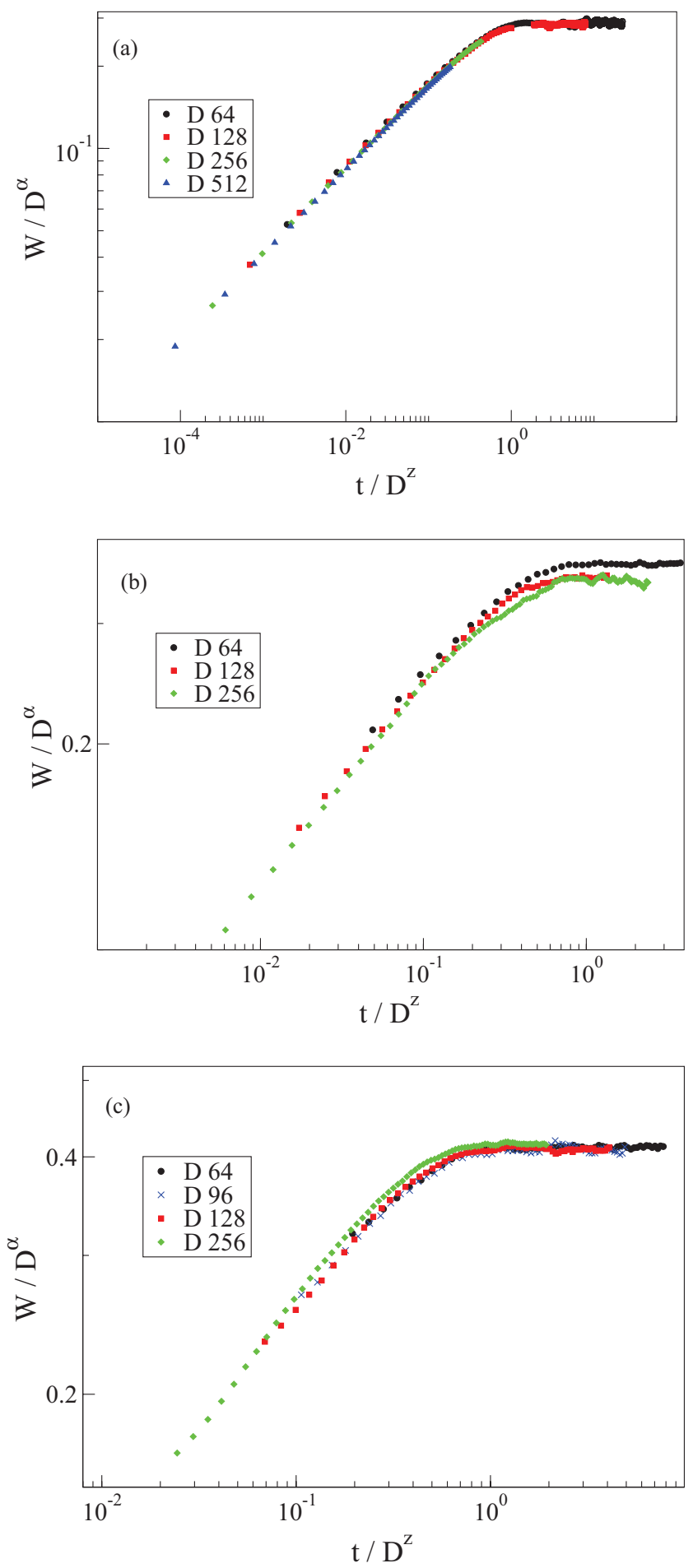

FIG. 6. (Color online) Log-log plots of the scaled width, $\frac{W}{D^{\alpha}}$ versus $\frac{t}{D^{z}}$, as it follows from Eq. (6), and applied to the data already shown in Fig. 4. The data collapse is achieved, in all cases, by using the exponents of the KPZ universality class, namely $\alpha=1 / 2$ and $z=3 / 2$. More details in the text.

Furthermore, the experimental and simulation geometry shown in Fig. 1 are also suitable for the evaluation of the density profiles of the growing cells, as depicted in Fig. 7(a), which show plots of the density profiles of cells $I_{1}, I_{2}$, and $M$ versus the axis perpendicular to the growth front (i.e., the $x$ axis in Fig. 1). 

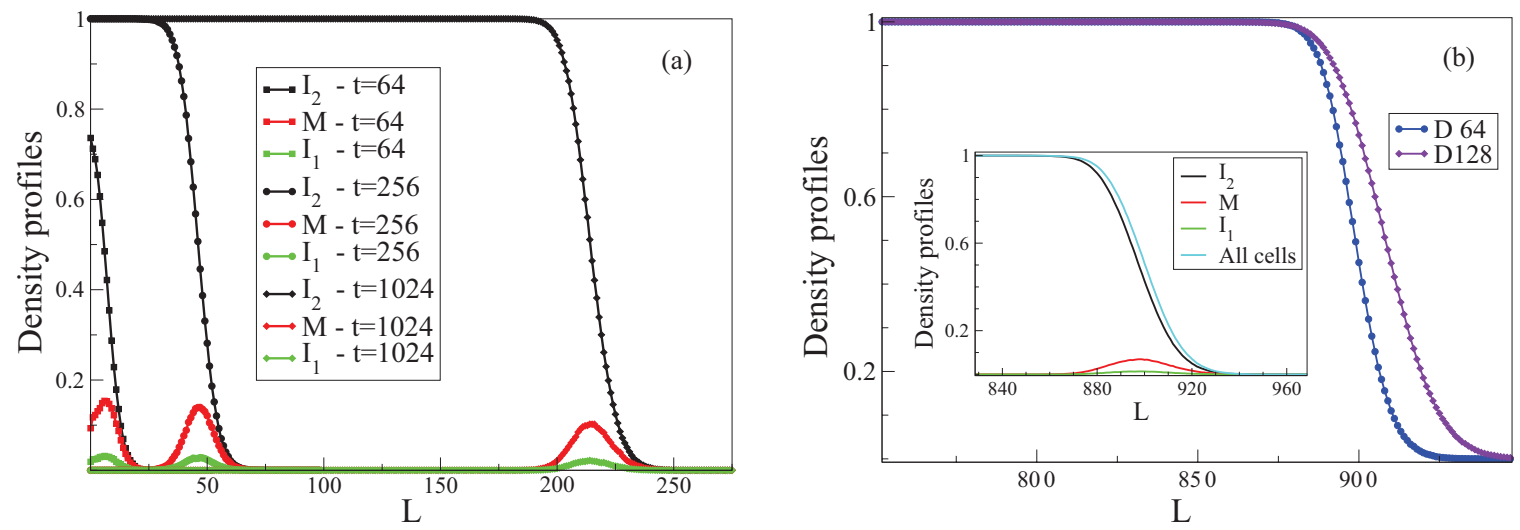

FIG. 7. (Color online) (a) Plots of the averaged density profiles of cells of types $I_{1}, I_{2}$, and $M$ as obtained for $P_{I}^{G}=0.10$ and samples of width $D=64$. The different types of cells are indicated. Measurements at times $t=64,256$, and 1024, respectively, from left to right. (b) Averaged density profiles of $I_{2}$ cells as obtained for samples of width $D=64$ and $D=128$ and for $P_{I}^{G}=0.10$. The inset shows that the density profile of all types of cells present in the culture is almost the same as the one corresponding to $I_{2}$ cells only. Results corresponding to all density profiles are averaged over 5000 realizations. More details in the text.

All these curves were obtained at different times and one observes the growth of compact cultures of $I_{2}$ cells with $\rho=1$ up to $h \simeq\langle h\rangle-W / 2$. Subsequently, and within the interfacial region, the profile decreases smoothly in an $S$-shaped fashion. The compact structure observed in the bulk of the culture is in agreement with the qualitative snapshot configuration shown in Fig. 2. Also, $M$ and $I_{1}$ cells exhibit "Gaussian-like" profiles and they are always close to the interface of the front. This scenery is consistent with the experimental observation that during tumor growth the maximum proliferation activity occurs at the growth interface [7,12-14]. On the other hand, Fig. 7(b) shows density profiles obtained for samples of different width. Here, one observes that the $S$-shaped tail of the profiles depends on $D$, in agreement with the fact that the interface width increases as $W_{s} \propto D^{\alpha}$ [cf. Eq. (8)]. Moreover, the inset of Fig. 7(b) shows that the difference between the density profiles of all types of cells and the one corresponding to $I_{2}$ cells only is almost negligible, a fact that we will use in order to support some theoretical calculations that are developed below.

The density profiles of $I_{2}$ cells shown in Fig. 7(a), or the profiles of all types of cells shown in the inset of Fig. 7(b), are nothing else but a microscopic (discrete) way of measuring the so-called traveling waves, which can be related to the solution of the Fisher-Kolmogorov (FK) equation [44]. It is worth mentioning that the density profiles are obtained by integrating (actually, by adding) the amount of cells distributed in $d=(1+1)$ dimensions along the vertical direction, cf. Figs. 1 and 2, so they correspond to $1 d$ projections of the mass of the culture. Therefore, they can well be described by the traveling waves that are solutions of the FK equation in $d=1$ dimension. In fact, the one-dimensional stochastic FK equation for the normalized local density cells, $u(x, t)$, reads

$$
\frac{\partial u}{\partial t}=D^{*} \frac{\partial^{2} u}{\partial x^{2}}+\alpha_{P} u(1-u)+\epsilon \sqrt{u(1-u)} \eta(x, t) .
$$

Here the cell mobility is accounted for the first term of the right-hand side with the diffusion constant $D^{*}$, while cell proliferation at a constant rate $\alpha_{P}$ is considered by means of the second term of the right-hand side. Also, we considered the effect of the noise (third term of the right-hand side), where $\eta(x, t)$ is a Gaussian white-noise process in space and time satisfying $\langle\eta(x, t)\rangle=0$ and $\langle\eta(x, t) \eta(y, s)\rangle=\delta(x-y) \delta(t-$ $s)$. We will refer to the coefficient $\epsilon$ as the noise strength, which one may think of as being proportional to $1 / \sqrt{N}$, where $N$ is the saturation population at a lattice site or in an appropriately defined correlation volume.

By neglecting the stochastic term, the FK equation has been used to describe some biological systems, e.g., the migration of brain tumor cells cultured in vitro [28], the dynamic behavior of bacterial populations [45], and so on. We have solved the FK equation by using standard integration methods (see, e.g., Fig. 8), which show results obtained by taking $\epsilon=0$ and

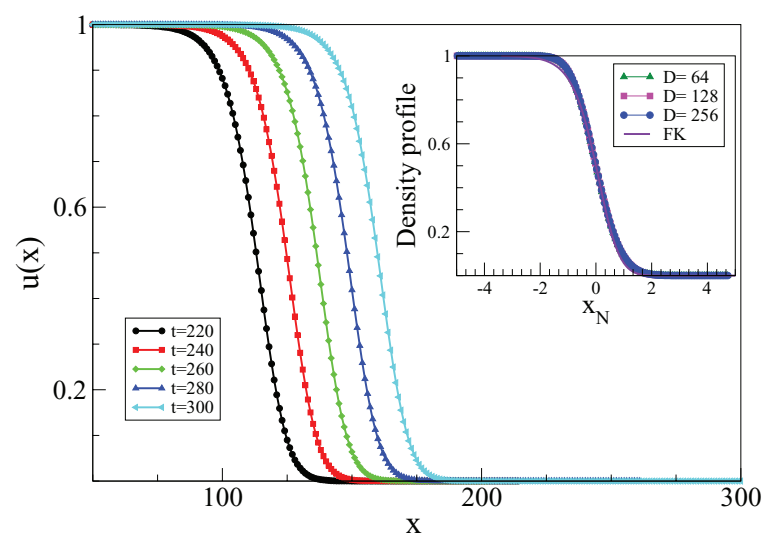

FIG. 8. (Color online) Density profiles obtained by solving Eq. (15) for $\alpha_{p}=0.1$ and $D^{*}=0.9$ in the absence of noise $(\epsilon=0)$. For the sake of comparison, in the inset we show results obtained for the discrete growth model (DGM) by taking different values of the sample width $D$ and $P_{I}^{G}=0.10$. Here, data collapse is achieved after proper normalization of the horizontal axis (see the text). The solid line corresponds to a solution of the Fisher-Kolmogorov (FK) equation (15) for $\alpha_{p}=0.1$ and $D^{*}=0.9$ in the absence of noise $(\epsilon=0)$. More details in the text. 
assuming the following initial condition:

$$
u(x, 0)=\left\{\begin{array}{lll}
1, & \text { if } & x \leqslant 0 \\
0, & \text { if } & x>0
\end{array} .\right.
$$

In order to compare our numerical results with simulations of the discrete model, we recall that, in the latter case, the $S$-shaped tail of the profiles depends on the length of the vertical integration range $(D)$ since it spreads around the half-density point, i.e., $u(\langle h\rangle)=1 / 2$, over a region given by $h \simeq\langle h\rangle \pm W / 2$, where $W$ is the $D$-dependent average width of the profile. Therefore, simulation results obtained by using different sample widths can be collapsed by properly normalization of the horizontal axis, e.g., by taking $x_{N}=$ $\{x-x(\langle h\rangle)\} / W$, as shown in the inset of Fig. 8. Here, we observe not only the excellent data collapse achieved but also that the universal collapsed curve is in remarkable agreement with the numerical solution of the FK equation. This result strongly suggests that the traveling waves of the FK equation can be identified with the (universal) collapsed curves of the discrete model, as obtained by proper normalization and for a suitable set of parameters, e.g., $D^{*}$ and $\alpha_{P}$.

As becomes evident, we postulated the FK equation for a single cell type, an assumption that is justified by the fact that the density profiles measured for our model and all types of cells are almost the same as the profiles corresponding to $I_{2}$ cells only [cf. the inset of Fig. 7(b)].

It is worth mentioning that the traveling waves propagate at constant velocity [44], in agreement with our simulation results shown in Fig. 2(a). With the aid of the profiles evaluated by using the FK equation in the absence of noise $(\epsilon=0)$, we determined the velocity of the traveling waves $\left(v_{\mathrm{TW}}\right)$, which is conceptually equivalent to the so-called growth velocity $\left(v_{g}\right)$ within the discussion context of the results shown in Fig. 3(b). The velocities evaluated for different values of $\alpha_{p}$ and by $D^{*}=1-\alpha_{p}$, in agreement with our simulation model that takes $P_{I}^{D}=1-P_{I}^{G}$, are shown in Fig. 9. As in the case of our results obtained by using the discrete growth model (DGM), $v_{\mathrm{TW}}$ exhibits a maximum just at $\alpha_{p}=1 / 2$, and for lower values of $\alpha_{p}$ (e.g., $\alpha_{p} \leqslant 0.2$ ) the behavior of $v_{\text {TW }}$ versus $\alpha_{p}$ is consistent with Eq. (13). Furthermore, the best fit of data yields $\gamma=0.48(3)$, in excellent agreement with the simulation results, namely $\gamma=0.47(4)$. The exponent $\gamma=1 / 2$, obtained by fitting the results of the FK equation, is expected since the exact result for the limit velocity is $v_{\mathrm{TW}} \sim \sqrt{D^{*} \alpha_{p}}$ [44], which for low values of $\alpha_{p}$, i.e., the limit $D^{*} \rightarrow 1$ (since $D^{*}=1-\alpha_{p}$ ), one has $v_{\mathrm{TW}} \sim \alpha_{p}^{1 / 2}$. However, the agreement obtained with the exponent corresponding to the numerical Monte Carlo simulations is in principle nontrivial at all and further supports our description of the density profiles by means of the FK equation. On the other hand, a direct comparison between our simulation and numerical results (cf. the inset of Fig. 9) shows a remarkable qualitative agreement.

Summing up, we conclude that, in the absence of noise, the FK equation provides a suitable mean-field description of the density profiles and propagation velocity, which is in full qualitative agreement with the simulation of the discrete growth model. Of course, a more quantitative fit is no longer possible due to the discrete versus continuous nature of time

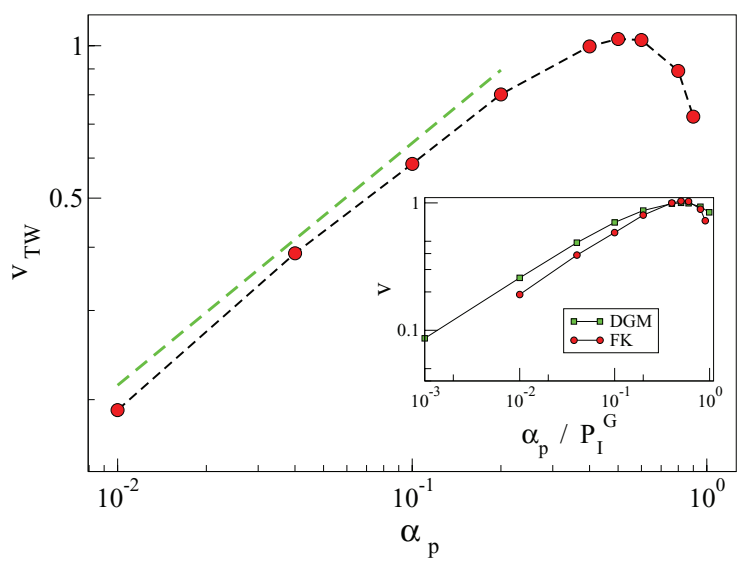

FIG. 9. (Color online) Log-log plots of the propagation velocity of the traveling waves $v_{\text {TW }}$ versus $\alpha_{p}$ as obtained from the profiles calculated by using the FK equation. The dashed line (slightly shifted up for the sake of clarity) has slope $\gamma=0.48$ (3), as follows from the best fit of the data obtained according to Eq. (13) and for $\alpha_{p} \leqslant 0.2$. The inset shows a comparison of the results obtained by using our discrete growth model [cf. Fig. 3(b)] and the solution of the FK equation. The data are normalized with respect to the maximum value of the velocity for the sake of comparison. More details in the text.

and space involved in the DGM and the FK equation, respectively.

We have already compared well-averaged simulation profiles with the solutions of Eq. (15) in the absence of noise. However, in most experimental situations it is only possible to measure the growth of a limited number of cultures or tumors, a fact that introduces stochastic ingredients and behavior into the results to be analyzed. In those cases, it is more convenient to work with the stochastic FK equation, i.e., with $\epsilon \neq 0$, in order to achieve a more realistic comparison (cf. Fig. 10). In fact, by shifting the horizontal axis so $u(x)=1$ for $x \simeq 0$ in order to rescale the time, one observes a rather good qualitative agreement between the solutions of the stochastic FK equation and the results of a single simulations of our proposed discrete growth model.

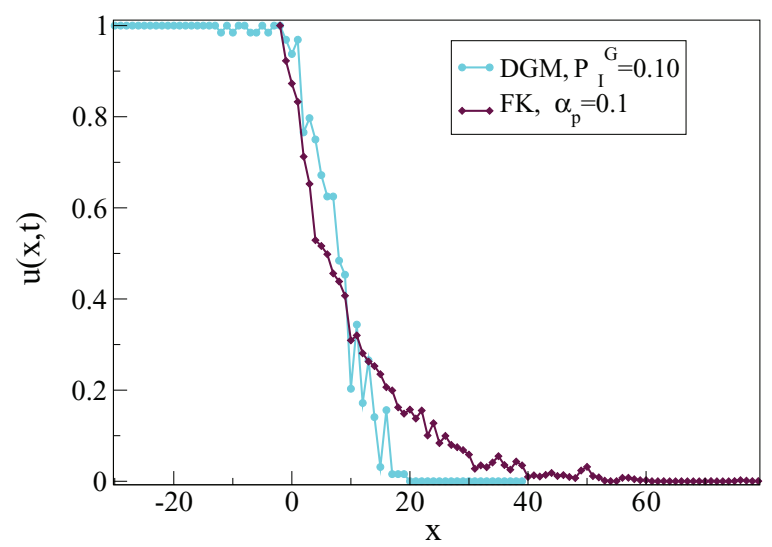

FIG. 10. (Color online) Density profiles obtained by solving the Fisher-Kolmogorov (FK) equation (15) for $\alpha_{p}=0.1$ in the presence of noise $(\epsilon=1)$. For the sake of comparison, we show Monte Carlo simulation results obtained for the discrete growth model (DGM) with $D=64$ and $P_{I}^{G}=0.10$. More details in the text. 


\section{CONCLUSIONS}

We presented and studied by means of extensive simulations a minimal discrete model for avascular tumor and cell culture growth, which incorporates the effects of excluded volume, cell proliferation, diffusion, and rotation. By performing a detailed finite-size scaling study complemented by a spectral analysis of the growth interface, we conclude that it is selfaffine and belongs to the KPZ universality class. In fact, this interface is described by the following critical exponents: the early time roughening is governed by the growth exponent $\beta=$ $1 / 3$, the stationary roughness of the interface is characterized by the exponent $\alpha=1 / 2$, while the correlations along the interface grow in time with a dynamic exponent $z=\alpha / \beta=$ $3 / 2$. This finding is in agreement with recent determinations of the interfacial properties of cultures of cancerous cells performed in vitro [21,22]. Also, the concentration profiles of the cells of the growing aggregate can be rationalized in terms of the propagation of traveling waves, which are derived from the discrete (microscopic) model and can be compared with the solutions of both the standard and the stochastic (macroscopic) Fisher-Kolmogorov equations.

It could be considered that the used geometry for the characterization of the interface, i.e., a front with linear symmetry, may be a limitation of the analysis since most in vitro experiments of tumor cell growth in Petri dishes are performed by starting with a "seed" culture placed in the center of the substrata, i.e., a geometry that leads to a circular pattern. However, by carefully comparing results obtained for the same kind of cells and experimental conditions but using both types of geometries (with linear and radial symmetry), it has been shown [21,22] that the KPZ nature of the interface remains unchanged. So, we have selected the linear geometry for our simulations not only based on that finding but also due to the fact that the linear geometry allows the straightforward evaluation of the complete set of exponents in order to determine the universality class of the interface (for a discussion of the experimental shortcomings involved in the determination of interfacial exponents by using radially growing pattern, see Ref. [46]).

Furthermore, we recognize that our minimal model neglects intracellular interactions based on cell signaling through, for example, growth factors or cytokines. Moreover, the mechanical properties of the cells (beyond the excluded volume) are disregarded. However, despite these simplifications, our model is capable of capturing the essential features of the interfacial properties of two-dimensional cultures of cancerous cells performed in vitro $[21,22,47]$. Certainly the characterization of the tumor growing interface properties in a three-dimensional culture or in an actual tumor is much more difficult. In fact, for its simplicity, our model is expected to be more appropriate for the description of two-dimensional cell cultures in vitro. In this way, as pointed out by Smolle et al. [34], in vitro systems are usually simpler and easy to study, but often they do not reflect the actual in vivo situation of tumors. However, the study of in vitro systems has some advantages as compared to their in vivo counterpart: relatively easy reproducibility and the possibility of performing dynamic studies of the culture evolution.

Therefore, while the processes involved in both tumor and cell culture growth are quite complex, we show that the complementary analysis of the results obtained by using both a minimal model for discrete growth and a simple continuous equation is capable of accounting for the universal features of the growth interface by capturing only a few, but the most relevant, underlying ingredients. In this way, the proposal and understanding of minimal models represent a topic of interest since they contribute to the characterization of a complex phenomenon. Of course, for a complete description of the tumor growth, a more complete model is necessary, but this is beyond the scope of the present work.

\section{ACKNOWLEDGMENTS}

We acknowledge financial support from CONICET, ANPCyT, and UNLP (Argentina). We thank Maria Ana Huergo and Miguel Pasquale for fruitful discussions about this work.
[1] P. Tracqui, Rep. Prog. Phys. 72, 056701 (2009).

[2] D. Ribatti, History of Research on Tumor Angiogenesis (Springer, Berlin, 2009).

[3] L. M. Sander and T. S. Deisboeck, Phys. Rev. E 66, 051901 (2002).

[4] E. Khain and L. M. Sander, Phys. Rev. Lett. 96, 188103 (2006).

[5] S. A. Menchón and C. A. Condat, Phys. Rev. E 78, 022901 (2008).

[6] M. Block, E. Schöll, and D. Drasdo, Phys. Rev. Lett. 99, 248101 (2007).

[7] D. Drasdo and S. Höhme, Phys. Biol. 2, 133 (2005).

[8] S. Torquato, Phys. Biol. 8, 015017 (2011).

[9] T. S. Deisboeck, Z. Wang, P. Macklin, and V. Cristini, Annu. Rev. Biomed. Eng. 13, 127 (2011).

[10] P. Ciarletta, L. Foret, and M. Ben Amar, J. R. Soc Interface. 56, 345 (2011).

[11] Y. Jiang, J. Pjesivac-Grbovic, C. Cantrell, and J. P. Freyer, Biophys. J. 89, 3884 (2005).
[12] E. Khain, C. M. Schneider-Mizell, M. O. Nowicki, E. A. Chiocca, S. E. Lawler, and L. M. Sander, Euro. Phys. Lett. 88, 2199 (2000).

[13] A. Brú, S. Albertos, J. L. Subiza, J. López García-Asenjo, and I. Brú, Biophys. J. 85, 2948 (2003).

[14] A. Giese, R. Bjerkvig, M. E. Berens, and M. Westphal, J. Clin. Oncol. 21, 1624 (2003).

[15] F. Family and T. Vicsek, J. Phys. A 18, L75 (1985).

[16] A. L. Barabasi and H. E. Stanley, Fractal Concepts in Surface Growth (Cambridge University Press, Cambridge, 1995).

[17] A. Bunde and S. Havlin, Fractals and Disordered Systems (Springer-Verlag, New York, 1991).

[18] J. J. Ramasco, J. M. López, and M. A. Rodríguez, Phys. Rev. Lett. 84, 2199 (2000).

[19] H. E. Stanley, Introduction to Phase Transitions and Critical Phenomena (Oxford University Press, Oxford, 1971).

[20] A. Brú, J. M. Pastor, I. Fernaud, I. Brú, S. Melle, and C. Berenguer, Phys. Rev. Lett. 81, 4008 (1998). 
[21] M. A. C. Huergo, M. A. Pasquale, A. E. Bolzán, A. J. Arvia, and P. H. González, Phys. Rev. E 82, 031903 (2010).

[22] M. A. C. Huergo, M. A. Pasquale, P. H. González, A. E. Bolzán, and A. J. Arvia, Phys. Rev. E 84, 021917 (2011).

[23] P. Meakin, J. Theor. Biol. 118, 101 (1986).

[24] M. Matsushita and H. Fujikawa, Physica A 168, 498 (1990).

[25] M. Poujade, E. Grasland-Mongrain, A. Hertzog, J. Jouanneau, P. Chavrier, B. Ladoux, A. Buguin, and P. Silberzan, Proc. Natl. Acad. Sci. USA 104, 15988 (2007).

[26] N. S. Gov, Proc. Natl. Acad. Sci. USA 104, 15970 (2007).

[27] M. Bindsschadler and J. L. McGrath, J. Cell Sci. 120, 876 (2007).

[28] E. Khain, M. Katakowski, S. Hopkins, A. Szalad, X. Zheng, F. Jiang, and M. Chopp, Phys. Rev. E 83, 031920 (2011).

[29] D. O. Morgan, The Cell Cycle, Principles of Control (New Science Press Ltd, London, 2007).

[30] B. Sapoval, M. Rosso, and J. F. Gouyet, J. Phys. (Paris) Lett. 46, L149 (1985).

[31] V. C. Chappa and E. V. Albano, J. Chem. Phys. 121, 328 (2004).

[32] M. T. Gastner, B. Oborny, D. K. Zimmermann, and G. Pruessner, Am. Nat. 174, E23 (2009).

[33] E. S. Loscar, N. Guisoni, and E. V. Albano, Eur. Phys. J. B 85, 60 (2012).

[34] J. Smolle and H. Stettner, J. Theor. Biol. 160, 63 (1990).

[35] M. Henkel and M. Pleimling, Theoretical and Mathematical Physics, Non-Equilibrium Phase Transitions, Volume 2, Ageing and Dynamical Scaling far from Equilibrium (Springer, New
York, 2010); P. Papon, J. Leblond, and P. H. E. Meijer, The Physics of Phase Transitions, Concepts and Applications, 2nd ed. (Springer, Berlin, 2006).

[36] E. V. Albano, M. A. Bab, G. Baglietto, R. A. Borzi, T. S. Grigera, E. S. Loscar, D. E. Rodriguez, M. L. Rubio Puzzo, and G. P. Saracco, Rep. Prog. Phys. 74, 026501 (2011).

[37] A. B. Kolton, A. Rosso, E. V. Albano, and T. Giamarchi, Phys. Rev. B 74, 140201(R) (2006).

[38] M. Eden, Proc. 4th Berkeley Symp. on Math. Statistics and Probability, Vol. 4 (University of California Press, Berkeley, 1961).

[39] D. Drasdo, R. Kree, and J. S. McCaskill, Phys. Rev. E 52, 6635 (1995).

[40] S. Das Sarma, Fractals 1, 784 (1993).

[41] J. Buceta and J. Galeano, Biophys. J. 88, 3734 (2005).

[42] A. Brú, S. Albertos, J. L. Subiza, J. López García-Asenjo, and I. Brú, Biophys. J. 88, 3737 (2005).

[43] S. F. Edwards and D. R. Wilkinson, Proc. R. Soc. London A 381, 17 (1982); F. Family, Physica A 168, 561 (1990).

[44] J. D. Murray, Mathematical Biology. II. Spatial Models and Biomedical Applications, 3rd ed. (Springer, New York, 2003).

[45] V. M. Kenkre and M. N. Kuperman, Phys. Rev. E 67, 051921 (2003).

[46] M. A. C. Huergo, Ph.D. thesis, Universidad Nacional de La Plata, Argentina, 2011.

[47] M. A. C. Huergo, M. A. Pasquale, P. H. González, A. E. Bolzán, and A. J. Arvia, Phys. Rev. E 85, 011918 (2012). 